\title{
Veterans and chronic pain
}

\section{Summary points}

1. Musculoskeletal problems are the commonest reason for medical discharge in all the British armed forces. By definition, these problems are chronic and resistant to treatment.

2. Pain is also common in veterans who have experienced severe injuries (polytrauma), often accompanied by post-traumatic stress disorder (PTSD) and traumatic brain injury (TBI) orpostconcussive syndrome.

3. In veterans seeking treatment for chronic pain, PTSD is common. There is also evidence for elevated levels of alcohol misuse in veterans who have been deployed to conflict. However, most veterans do not have pain, PTSD or alcohol problems.

4. Pain clinicians would benefit from training in meeting veterans' needs, in order to promote their engagement and successful treatment. This should include countering stereotypes, information about the military and support for the assessment and onward referral of PTSD and alcohol problems.

\section{Keywords}

Back pain, chronic pain, headache, low back pain, pain management, phantom limb

\section{Introduction}

Combat operations in Afghanistan and Iraq have resulted in a steady flow of casualties, with over 6500 British personnel admitted to field hospitals in Afghanistan as of January 2013.1 As a consequence, there is substantial government and media interest in the health of veterans. Military trauma care has improved, both in the field and in the UK, such that servicemen and women are surviving severe injuries that would have proved fatal in previous conflicts. Improved body armour has also increased survival rates. ${ }^{2}$ Thus, there are an increasing number of servicemen and women who will go on to survive with the long-term consequences of serious injury (i.e. amputation, head injury, pain). In the USA, veterans are treated in the Veterans Affairs (VA) system, a dedicated and extensive network of 1700 nationwide treatment facilities. However, in most other countries, veterans will be treated in the public health system after discharge. American data suggest a high rate of persisting pain in veterans seeking healthcare treatment, with moderate to severe pain intensity identified in $28 \%$ of
US veterans seeking healthcare in a chart review. ${ }^{3}$ Pain appears to have a unique negative impact on the physical functioning of veterans, above and beyond variables such as depression and post-traumatic stress disorder (PTSD). ${ }^{3}$ This paper addresses this growing issue, and focuses on the treatment of chronic pain in veterans; we will emphasise the treatment of British veterans, but refer to international data, particularly that gathered in the VA system in the USA.

Although there has been an emphasis on severe combat injury, this may not be representative of the range of injury types and causes in the military. For

Bath Centre for Pain Services \& Department of Psychology, University of Bath, Bath, UK

\section{Corresponding author:}

Jeremy Gauntlett-Gilbert, Bath Centre for Pain Services, Royal National Hospital for Rheumatic Diseases NHS Foundation Trust, Upper Borough Walls, Bath BA1 1RL, UK.

Email: Jeremy.gauntlett-gilbertQrnhrd.nhs.uk 
example, there are many training injuries, and many casualties have experienced the military equivalent of a 'work place injury', whether in a combat zone or not on deployment. Military life in all services requires hazardous activity, such as operating heavy machinery in unpredictable environments. Accidents on land, in the air and at sea are inevitable. The importance of this broader focus is made clear by data from an American forward surgical team in Afghanistan; $42 \%$ of their work was non-combat injuries, with under half of their patients $(49 \%)$ having battle injuries. ${ }^{4}$ British data indicate that $68 \%$ of Afghanistan field hospital admissions have been for disease or non-battle injuries. ${ }^{1}$ Specifically, lower back pain is not necessarily associated with the physical aspects of combat or infantry load-carrying; a study of 37,000 US Marines with back pain (all having been deployed to Iraq), indicated the highest rates in service/supply and electrical/mechanical trades, rather than infantry. ${ }^{5}$ However, there are no equivalent data for a British population. Therefore, this paper reviews existing data that may clarify the broad range of chronic pain problems in veterans being treated in the public health system.

\section{Musculoskeletal pain and musculoskeletal injury}

Several sources of data point to high rates of musculoskeletal pain in veterans and serving military personnel. The best-conducted surveys of veterans' overall health investigated the experiences of Gulf War veterans. Kang et al. ${ }^{6}$ surveyed 11,000 American Gulf War veterans; the most commonly self-rated 'severe' healthcare problem (amongst all possible problems) was back pain (17\%), followed by joint pain (15\%). ${ }^{6}$ Unwin et al. ${ }^{7}$ surveyed 4000 British Gulf War veterans six years after deployment, and also found that back pain was the most common self-reported health problem at $36 \%$ (again, amongst all possible health problems; this reported rate is likely to be higher as there was no judgement of 'severity'). ${ }^{7}$ These high rates of back and joint pain are not purely due to Gulf War deployment, as they are mirrored in comparable non-Gulf veteran cohorts. Unwin et al. ${ }^{7}$ also surveyed a similar-sized cohort deployed to Bosnia and another cohort deployed to neither (called the 'Era' group). Back pain was also the most commonly reported problem (Bosnia, 24\%; Era, $28 \%$ ). Thus, back pain is common in veterans who have been operationally deployed and in those who have not, and so is likely to represent a key healthcare need of veterans. However, as there are high rates of back pain in the general population, it is impossible to know whether these high prevalences are particularly related to military service. Direct comparisons of pain prevalence in veterans versus age-matched civilian controls have not been carried out.

Medical discharge statistics offer another source of data on the health needs of veterans. British data indicate that musculoskeletal problems are by far the most common category for medical discharge. In 2011, musculoskeletal problems accounted for $57 \%$ of naval medical discharges, with $60 \%$ in the Army and $57 \%$ in the Royal Air Force; overall, 59\% of all medical discharges were coded as musculoskeletal problems. ${ }^{8}$ Women are more likely to be discharged with musculoskeletal injuries than men. ${ }^{9}$ The context of medical discharge provides further information. In general, musculoskeletal rehabilitation services in the British armed services are of high quality. For example, at a regional rehabilitation unit, a serving man or woman could receive a three-week, full-time residential rehabilitation programme, staffed with specialist medics, physiotherapists and exercise rehabilitation instructors. Clearly, this is more intensive and complete than most British public-health primary and secondary rehabilitation services. Thus, for a serviceman or woman to have reached medical discharge, musculoskeletal problems must have been (1) long-standing, (2) functionally interfering (or they would not have led to discharge) and (3) refractory to good-quality treatment. It is likely that a proportion of these problems will later manifest as chronic pain in veterans. The degree of functional impairment associated with medical discharge is not clear. For example, an injury that prevents an individual serving as a Royal Marine may present few barriers to them engaging in a more sedentary civilian job. However, a key study examined a group of American Iraq and Afghanistan veterans attending a health screening clinic. ${ }^{3}$ Despite the fact that most of them reported 'good' or 'fair' health (90\%), chronic widespread pain was common (29\%) and had a unique role in predicting poorer physical role functioning, independent of comorbid mental health problems. British data on the severity of veterans' health problems, or associated disability, are not available. Publicly available War Pensions data indicate the numbers of veterans claiming compensation for disability, but make no record of the cause of their impairments.

\section{Severe injury and polytrauma}

Although musculoskeletal problems may be most prevalent in military populations, the multiple medical and psychosocial needs of severely combat-injured servicemen and women demand attention. Surviving 'polytrauma' - injury to more than one body system or organ - is becoming increasingly common and the 
number of UK military amputees is increasing. For example, in 2007 and 2008, 45 amputations were recorded in Afghanistan; in 2010 and 2011 this figure had increased to $146 .{ }^{10}$ Polytrauma is frequently caused by blast injury, most commonly by improvised explosive devices, mortars and rocket-propelled grenades. Military medical services have now gained great experience in the treatment of people surviving polytrauma, and according to Sayer et $\mathrm{al}^{2}$ ' the frequency of pain is particularly remarkable and supports the development of pain management protocols for patients with polytraumatic combat injuries' (p. 168). ${ }^{2}$ In a chart review of 188 American polytrauma patients from Iraq and Afghanistan, pain was the second most common focus of rehabilitation ( $83 \%)$; only cognitive problems were more prevalent $(88 \%)$ and pain was also more frequent than mental health problems $(61 \%){ }^{2}$

Researchers and clinicians in the USA refer to a characteristic 'polytrauma clinical triad' of traumatic brain injury (TBI), chronic pain and PTSD. In a series of 340 patients treated at VA polytrauma centres, comorbidity between these diagnoses was vastly more common than seeing any one of them alone. ${ }^{11}$ TBI leads to a range of post-concussional symptoms, including cognitive dysfunction, fatigue and irritability. However, there is also a high rate of headache amongst polytraumatised veterans with TBI. A headache frequency of $63 \%$ has been found in this population, and $33 \%$ of veterans with mild TBI in a polytrauma centre required referral to a neurologist for assessment and treatment of their headaches. Headaches are more common in patients who have experienced penetrating head injuries (TBI and headache studies reviewed in Gironda et al., 2009), with most post-traumatic headaches classifiable as tension-type headaches. ${ }^{12}$

Veterans with polytrauma also experience back pain $\left(58 \%{ }^{11}\right)$ and phantom limb pain. Back pain is unsurprising, given the nature of blast injury. Explosions cause damage by an initial pressurised shock wave, by fragments and shrapnel, and also by the 'blast wind' that can throw the casualty with extreme force against a range of surfaces. The latter mechanism is a candidate for causing back and neck pain. Phantom limb pain following amputation is also a risk for individuals with polytrauma; $77 \%$ of a sample of 30 military traumatic amputees experienced phantom limb pain, with $68 \%$ receiving some form of treatment for this. ${ }^{13}$

The experience of the 'polytrauma clinical triad' has led to authors from the VA system suggesting pathways for treatment that respect this comorbidity, with a sense of this broad symptom complex as a 'final common pathway' for a range of conditions, injuries, risks and exposures. ${ }^{14}$ It certainly seems necessary for civilian healthcare providers to prepare to treat this combination of difficulties. It is also important to appreciate the context within which such injuries occur. For example, the combat experience can include experiences of chronic stress, sleeplessness, exposure to environmental hazards (e.g. vehicle fumes, smoke, sand, dust), heat stroke, acoustic trauma, multiple vaccinations and use of anti-malaria pills and insecticides. ${ }^{14}$ Sayer et al. ${ }^{2}$ compared polytrauma caused by blast injury with that from other causes. ${ }^{2}$ They concluded that, while blast injuries did not cause globally poorer treatment response, they were associated with a particular constellation of injuries. These included greater rates of eye, oral and maxillofacial injuries, along with higher rates of penetrating brain injury, PTSD and auditory impairments. Some of these are likely to become life-long legacies of injury.

\section{Pain and comorbid mental health problems}

The above data on polytrauma emphasise the need to anticipate comorbidity in chronic pain presentations. Civilian samples have always shown high rates of anxiety and depression in people with chronic pain. However, military service may predispose veterans to specific mental health problems, such as PTSD, and military recruits may not be drawn from a typical section of the UK population, bringing their own preexisting vulnerabilities and strengths. Data on likely comorbidities are essential for service planning.

Fear et al. ${ }^{15}$ rigorously surveyed a large sample of British servicemen and women who had been either deployed or not deployed to Iraq and Afghanistan. ${ }^{15}$ In this anonymised self-report survey, rates of PTSD were low $(4.0 \%)$ with alcohol misuse $(13.0 \%)$ and common mental disorders (19.7\%) much more prevalent. Being deployed into a theatre of operations had the effect of increasing alcohol misuse (deployed, 15.7\%; nondeployed, $10.9 \%$ ), and being involved in combat increased rates of PTSD $(6.9 \%$, versus $3.6 \%$ in a combat support sample). These data indicate that PTSD rates after deployment are reassuringly low, but that future health service provision should prepare for PTSD and alcohol problems, given the large numbers deployed to Iraq and Afghanistan over the years $(>100,000)$. It is not known how rates of distress will change with increased time since deployment. Fear et al. ${ }^{15}$ recorded a small increase in the reporting of PTSD over time, and delayed-onset PTSD accounts for $38 \%$ of military cases. ${ }^{16}$

Veterans seeking treatment are likely to have higher rates of emotional distress than a general cohort sample, and chronic pain and PTSD are likely to co-occur. For example, Shipherd et al. ${ }^{17}$ studied veterans presenting for PTSD treatment, and found a $66 \%$ rate of physician-diagnosed chronic pain. The most common 
pain diagnoses were lower back pain, pain associated with a chronic condition (e.g. diabetes) and osteoarthritis. ${ }^{17}$ Conversely, Otis et al. ${ }^{18}$ studied 149 veterans in a psychology pain management programme. Diagnostic criteria for PTSD were met in $49 \%$ of cases using the PTSD Checklist - Military version (PCL-M). ${ }^{18}$ Two key theories have been proposed to account for the high comorbidity between PTSD and chronic pain. The 'shared vulnerability' hypothesis proposes that an underlying trait, such as anxiety sensitivity (the fear that anxiety symptoms are harmful) predisposes patients to both pain and PTSD. Alternatively, the 'mutual maintenance' hypothesis suggests that pain may trigger traumatic memories, and traumatic hyperarousal may worsen pain. Patterns of behavioural avoidance may then mutually maintain both pain and PTSD. This hypothesis is supported by results showing that veterans with comorbid pain and PTSD show higher levels of catastrophic thinking and perceived lack of control over pain. ${ }^{19}$ These data suggest that combined PTSD and pain treatment may be possible. This hypothesis is currently being tested by the American military; a study run by the South Texas Research Organizational Network Guiding Studies on Trauma and Resilience (STRONG STAR) includes four arms: (1) pain management only, (2) PTSD treatment only, (3) combined PTSD and pain treatment, and (4) treatment as usual. ${ }^{20}$ The results of this research have not yet been published.

\section{Recommendations for service planning and treatment}

The data reviewed above indicate that British veterans will commonly present to pain services with musculoskeletal pain, particularly back pain. Those seeking treatment are likely to have high rates of comorbid emotional problems, similar to civilian populations, but with the potential for particularly high rates of PTSD and alcohol problems. Encouragingly, most pain services, and particularly multidisciplinary pain management programmes, have highly applicable skills for this veteran population. Developments in the US military have involved adapting interdisciplinary chronic pain programmes for military settings. ${ }^{20}$ There will also be a minority of patients who have experienced severe injuries and have multiple chronic problems. Current services may be less ideally configured for these patients. Here, we examine ways to serve the veteran population best. The NHS doubtless has lessons to learn from military rehabilitation clinicians. However, the public health system will be challenged by treating veterans 5, 10 and 20 years after discharge. In the UK, military medical and rehabilitation services have no experience of these populations, as they do not treat patients after discharge. There are no definitive British population data on veterans' health needs, although certain ex-services charities collect data on their contacts (e.g. Combat Stress for mental health problems in the UK). Thus, these recommendations are provisional and anecdotal.

\section{Staff competencies}

In the USA, veterans are treated by VA clinicians who have a guaranteed minimum knowledge of military service and its legacies. Elsewhere, where veterans are treated in public health systems, there is no reason to believe that pain clinicians have any of this relevant knowledge. However, all pain clinics will currently be treating veterans, whether they are aware of it or not. Pain clinicians have a duty to attempt to understand this population, just as much as any other demographic minority with specific needs. Two competencies seem essential; the ability to anticipate and handle traumatic disclosure, and some knowledge of military 'cultures'.

As seen above, rates of PTSD are high in veterans seeking help for chronic pain. Even where diagnosable PTSD is not present, sub-clinical traumatic distress may be significant, and individuals may benefit greatly from disclosing trauma to a professional. An in-depth study of 23 US veterans suggested that they appreciated direct, informal questioning about trauma using open-ended questions. ${ }^{21}$ Disclosure was easier when the clinician did not rush, ensured privacy and made an attempt to ease the patient's sense of shame. Civilian pain clinicians can be trained in recognising PTSD symptoms, normalising distress and reducing shame, and in the supportive interviewing skills that aid openness. This will usually require access to psychological supervision, in order to maintain skills, discuss mental health issues and prevent 'burnout' where traumatic disclosures are frequent or distressing.

NHS staff can also benefit from 'cultural' knowledge about the armed services, and from information that counters media stereotypes of veterans. For example, from broadcast and print media it is easy to conclude that veterans have high rates of PTSD, are largely amputees or are largely flourishing with an appetite for adventurous training. None of these assumptions can be substantiated. Even positive stereotypes, such as news stories of amputees climbing mountains, can discourage veterans who cannot, or do not wish to, access such activities. 'Cultural' training for NHS staff might include (1) knowledge of the range of branches and trades in the military (including the predominance of non-combat roles), (2) some idea of rank structures, (3) knowledge of military rehabilitation facilities and approaches, (4) the discharge process and its impact, (5) 
family issues in the military and (6) services cultures around physical exercise. The Scottish Association for Mental Health's guide entitled 'Life Force' is a helpful reference for clinicians. ${ }^{22}$

\section{Service design and planning}

Pain management programmes (PMPs) and multidisciplinary pain clinics have expertise in treating comorbid pain and emotional problems, and in addressing long-term problems with life and role functioning. These skills are relevant for veterans, with the traditional staffing mixture of psychology, physiotherapy and occupational therapy being appropriate. The ability to assess for PTSD and alcohol problems will be essential, and assessing clinicians will need the experience to know the level of comorbidity that can be treated on a PMP, or the level of comorbidity which requires prior treatment by other services, both in the health service and third sector (e.g. Combat Stress). Thus, a minimum level of psychology staffing is essential.

Health services should develop their awareness of veterans' charities and welfare organisations. Whilst veterans face specific challenges due to their history of service, they can also access more support than civilians with comparable disabilities. This can include counselling, family support, help to return to work and financial grants for household needs. Organisations provide specialist support for certain conditions, such as the British Limbless Ex-Servicemen's Association (BLESMA) for amputees and Blind Veterans UK for visual impairment. The best points of access for British veterans are the Confederation of Service Charities (COBSEO, www. cobseo.org.uk) and the Service Personnel and Veterans Agency (SPVA, www.veterans-uk.info).

Services that wish to identify and respond specifically to their veteran patients will need to make a decision about whether to 'mainstream' veterans into standard services or to create veterans-only interventions. In the USA, this decision is already made by the existence of the veterans-only VA system. Elsewhere, it is an important choice, particularly for group-based PMPs, where veterans-only groups would be easy to arrange. A study on identity change in traumatised or injured British veterans identified a range of themes around military identity, such as 'estrangement' from civilian society and a 'rejection of civvy street'. ${ }^{23}$ It is unclear whether these are best addressed by exposure to civilian life or by continued contact with ex-service personnel. Veterans-only groups and interventions offer the opportunity for social support from others with a similar background. However, they can limit the clinical agenda, where treatment goals emphasise integration into civilian life, adjustment to the end of a military career or developing new approaches to physical exercise. Anecdotally, we have found that individuals and organisations more closely allied to serving personnel favour veterans-only provision. However, charities engaged in long-term support for veterans adapting to civilian life also emphasise the benefits of 'mainstreaming'.

\section{Clinical and treatment issues}

Pain services need to find out and record whether patients are veterans. Minimum data should include veterans' status and whether the pain problem is service-related. Assessment of veterans should include their service history. Questioning could include 'trade' (e.g. engineer, submariner, cook); length of service; deployment; exposure to trauma; reason for and rank at discharge; family status; current contact and support from ex-services organisations, including war pensions issues. Physical rehabilitation is a particular challenge. In general, veterans in treatment have an extensive history of exercise, and have a high level of conviction about a particular approach to exercise that has worked well for their physical conditioning in the past (though usually when much younger, and in better health). Anecdotally, veterans appear to struggle with pacing themselves, acknowledging their limitations and paying mindful attention to physical sensations.

\section{Summary}

There is good reason to believe that veterans will regularly attend health services for the treatment of their chronic musculoskeletal pain. An important minority will also need pain treatment whilst living with the longterm consequences of severe injury. These patients have the capacity to be well treated in multidisciplinary pain clinics and PMPs. However, staff training around veterans' issues is essential, and appropriate provision and support for the treatment of comorbid PTSD and alcohol problems is essential.

\section{Acknowledgements}

The authors would like to thank the organisers and members of the NHS South West Armed Forces Forum for providing a context for the discussion of these issues.

\section{Conflict of interest}

The author declares that there is no conflict of interest.

\section{Funding}

Authors are funded by the Royal National Hospital for Rheumatic Diseases NHS Foundation Trust. 


\section{References}

1. Ministry of Defence. Op herrick casualty and fatality tables. Available at: http://www.mod.uk/DefenceInternet/ AboutDefence/CorporatePublications/DoctrineOperationsandDiplomacyPublications/OperationsInAfghanistan/ OpHerrickCasualtyAndFatalityTables.htm (2012, accessed 15 February 2013).

2. Sayer NA, Chiros CE, Sigford B, et al. Characteristics and rehabilitation outcomes among patients with blast and other injuries sustained during the global war on terror. Arch Phys Med Rehabil 2008; 89: 163-170.

3. Helmer DA, Chandler HK, Quigley KS, et al. Chronic widespread pain, mental health, and physical function in OEF/OIF veterans. Pain Med 2009; 10: 1174-1182.

4. Shen-Gunther J, Ellison R, Kuhens C, et al. Operation enduring freedom: trends in combat casualty care by forward surgical teams deployed to Afghanistan. Mil Med 2011; 176: 67-78.

5. MacGregor AJ, Dougherty AL, Mayo JA, et al. Occupational correlates of low back pain among US Marines following combat deployment. Mil Med 2012; 177: 845-849.

6. Kang HK, Mahan CM, Lee Y, et al. Illnesses among United States veterans of the Gulf War: a populationbased survey of 30,000 veterans. F Occup Environ Med 2000; 42: 491-501.

7. Unwin C, Blatchley N, Coker W, et al. Health of UK servicemen who served in the Persian Gulf War. Lancet 1999; 353: 169-178.

8. Ministry ofDefence.UKdefence statistics 2011. Available at: www.dasa.mod.uk/modintranet/UKDS/UKDS2011/pdf/ ukds.pdf (2011, accessed 1 June 2012).

9. Geary KG, Irvine D and Croft AM. Does military service damage females? An analysis of medical discharge data in the British armed forces. Occup Med 2002; 52: 85-90.

10. Ministry of Defence. Quarterly Afghanistan and Iraq amputation statistics. Available at: www.dasa.mod.uk (2013, accessed 15 February 2013).

11. Lew HL, Otis JD, Tun C, et al. Prevalence of chronic pain, posttraumatic stress disorder and persistent concussive symptoms in OIF/OEF veterans: polytrauma clinical triad. F Rehabil Res Dev 2009; 46: 697-702.
12. Gironda RJ, Clark ME, Ruff RL, et al. Traumatic brain injury, polytrauma, and pain: challenges and treatment strategies for the polytrauma rehabilitation. Rehabil Psychol 2009; 54: 247-258.

13. Ketz AK. The experience of phantom limb pain in patients with combat-related traumatic amputations. Arch Phys Med Rehabil 2008; 89: 1127-1132.

14. Uomoto JM and Williams RM. Post-acute polytrauma rehabilitation and integrated care of returning veterans: toward a holistic approach. Rehabil Psychol 2009; 54: 259-269.

15. Fear NT, Jones M, Murphy D, et al. What are the consequences of deployment to Iraq and Afghanistan on the mental health of the UK armed forces? A cohort study. Lancet 2010; 375: 1783-1797.

16. Andrews B, Brewin CR, Stewart L, et al. Comparison of immediate-onset and delayed-onset posttraumatic stress disorder in military veterans. F Abnorm Psychol 2009; 118: 767-777.

17. Shipherd JC. Keyes M, Jovanovic T, et al. Veterans seeking treatment for posttraumatic stress disorder: what about comorbid chronic pain? F Rehabil Res Dev 2007; 44: 153-166.

18. Otis JD, Gregor K, Hardway C, et al. An examination of the co-morbidity between chronic pain and posttraumatic stress disorder on US veterans. Psychol Serv 2010; 7: $126-135$.

19. Alschuler KN and Otis JD. Coping strategies and beliefs about pain in veterans with comorbid chronic pain and significant levels of posttraumatic stress disorder symptoms. Eur F Pain 2012; 16: 312-319.

20. McGeary D, Moore M, Vriend CA, et al. The evaluation and treatment of comorbid pain and PTSD in a military setting: an overview. F Clin Psychol Med Settings 2011; 18: $155-163$.

21. Jeffreys MD, Leibowitz RQ, Finley E, et al. Trauma disclosure to health care professionals by veterans: clinical implications. Mil Med 2010; 175: 719-724.

22. Scottish Association for Mental Health. Life Force: a practice guide for working with military veterans. Available at: http:/www.samh.org.uk/media/78014/life_force. pdf (2009, accessed 1 June 2012)

23. Brewin CR, Garnett $R$ and Andrews B. Trauma, identity and mental health in UK military veterans. Psychol Med 2011; 41: 1733-1740. 